\title{
Thin-walled compliant mechanism component design assisted by machine learning and multiple surrogates
}

\author{
Kai Liu', Andres Tovar ${ }^{2}$, Emily Nutwell ${ }^{3}$, Duane Detwiler ${ }^{3}$ \\ ${ }^{1}$ Purdue University ${ }^{2}$ Indiana University - Purdue University Indianapolis ${ }^{3}$ Honda R\&D Americas Inc.
}

\begin{abstract}
CITATION: Liu, K., Tovar, A., Nutwell, E., and Detwiler, D., "Thin-Walled Compliant Mechanism Component Design Assisted by Machine Learning and Multiple Surrogates," SAE Technical Paper 2015-01-1369, 2015, doi:10.4271/2015-01-1369.
\end{abstract}

Copyright $(2015$ SAE International

\begin{abstract}
This work introduces a new design algorithm to optimize progressively folding thin-walled structures and in order to improve automotive crashworthiness. The proposed design algorithm is composed of three stages: conceptual thickness distribution, design parameterization, and multi-objective design optimization. The conceptual thickness distribution stage generates an innovative design using a novel one-iteration compliant mechanism approach that triggers progressive folding even on irregular structures under oblique impact. The design parameterization stage optimally segments the conceptual design into a reduced number of clusters using a machine learning K-means algorithm. Finally, the multiobjective design optimization stage finds non-dominated designs of maximum specific energy absorption and minimum peak crushing force. The proposed optimization problem is addressed by a multiobjective genetic algorithm on sequentially updated surrogate models, which are optimally selected from a set of 24 surrogates. The effectiveness of the design algorithm is demonstrated on an S-rail thin-walled structure. The best compromised Pareto design increases specific energy absorption and decreases peak crushing force in the order of $8 \%$ and $12 \%$, respectively.
\end{abstract}

\section{Introduction}

Vehicle crashworthiness relies heavily on the energy absorbing capabilities of plastically deformable progressive crushing zones located at the front and rear end of the vehicle's body. In commercial vehicles, progressive crushing zones are comprised of thin-walled structures arranged in the form of hollow tubes, which are ultimately responsible for managing impact energy in the event of a collision. Thin-walled components are highly formable, structurally sound, and capable of sustaining axial collapse mode. This collapse mode, also known as progressive folding, is achievable by tubes of uniform thickness under axial load [3]. Geometric and material discontinuities as well as strain-rate effects and oblique impact promote a less desirable bending collapse mode. This low-energy collapse mode, also known to as Euler-type buckling, reduces the component crashworthiness and increases the risk of damage and intrusion in other zones of the vehicle [4].

Page 1 of 13
In order to mitigate this undesirable Euler-type buckling, vehicle structural designers have incorporated design features such as crush initiators in the form of cutouts, dents, and stiffeners [5-9]. Another alternative, which can be combined with crush initiators, is the use of cellular materials (honeycombs) and foam fillers of uniform and functionally graded density [10-13].

The integration of design optimization methods, which potentially reduce the design cycle time and increase the effectiveness of the structural component $[14,15]$, is hindered by two technical challenges. The first challenge is the conceptual design generation: the best type of crush initiator, cellular pattern, or foam density distribution, is unknown and the designer has to test several configurations before committing to a design that can be further parameterized and optimized. The second challenge is the nonlinearity of the crash computational model and the consequent computational cost of the simulation, which makes it impractical to use traditional optimization methods.

To systematically address the conceptual design generation of crashworthy structures, researchers have explored the use of topology optimization methods (material distribution) [16] using analytical approximations of the sensitivity coefficients. Methods based on linear implicit finite element analysis, such as equivalent static loads (ESL) [17, $18]$, or partially non-linear implicit methods $[19,20]$ are numerically efficient since sensitivity coefficients can be obtained; however, these methods are unable to capture all the relevant aspects of the transient crash event. Truly non-linear explicit methods have been applied using heuristic methods leading to innovative and useful conceptual designs [21-27]. Some of the main developments have been achieved by the hybrid cellular automaton (HCA) method proposed by the corresponding author and collaborators [24-27]. The premise of the method is that high-energy absorbing structures can be synthesized by uniformly distributing the internal energy density in a voxel-based discretized design domain.

Recently, our research group has proposed the use of HCA topometry (thickness distribution) methods for compliant, plastically deformable structures to support the generation of conceptual design for progressively folding thin-walled components including S-rails [28]. This method follows the design principles of compliant mechanisms and their ability to transfer force and displacement from a given input

This is the author's manuscript of the article published in final edited form as: 
to a desired output port [29]. The conceptual thickness distribution, usually achieved under one hundred crash simulations, has proven useful as benchmark design for progressive folding; however, this conceptual design does not depict particularly high specific energy absorption and low peak crushing force.

In this paper, we introduce a new approach in the conceptual design generation of thin-walled structures and further multi-objective optimization. The proposed conceptual design, based on HCA topometry, is obtained with a single crash simulation. Further, data clustering is implemented to reduce the dimension of the design space and allow structural optimization. Even though data clustering is commonly used in data mining and machine learning, our paper presents one of the first applications in structural design. Finally, we introduce a multi-objective global optimization method based the application of genetic algorithms on sequentially updated surrogate models. These models are selected based on a study of 24 surrogates. The method is demonstrated on the design of S-rail structures. The results demonstrate remarkable improvements in all crashworthiness indicators.

The body of the paper is organized in three main sections: conceptual design generation, design parameterization, and multi-objective optimization. A summary and discussion of the results are presented in the final section.

\section{Conceptual Design}

Thin-walled S-rail structures are the central components of vehicle's progressive crushing zones and absorb the highest amount of the kinetic energy during a frontal or rear collision. Various investigations on S-rail structures have addressed the effect of triggering mechanisms through changes in the cross section $[30,31]$. Typically, the design objective is to find a feasible geometry capable of sustaining high specific energy absorption through the structure's progressive folding.

Our work addresses the thin-walled structural design problem in two stages: conceptual design generation and structural optimization. The conceptual design generation, summarized in this section, consists on finding the thickness distribution that maximizes the displacement of a trigger (output port) for the crash load condition (input port). This problem, which corresponds to the one of a compliant mechanism, has been previously introduced by our group [27-29] and is summarized here. One outstanding difference between our previous contributions and this work is the reduction in the number of iterations from about one hundred to only one.

\section{Problem statement}

The design of a compliant mechanism using topometry optimization finds the thickness distribution that maximizes the displacement at the output port [32] or the mutual potential energy [33] subjected to an equality mass constraint. For a thin-walled S-rail structure as shown in Figure 1, the input ports are prescribed at the contact nodes with a rigid wall. The output ports correspond to the desired buckling trigger locations. Such locations may be prescribed according to the designer's criterion. However, they are naturally assigned by the wavelength $\lambda$ of the progressive buckling corresponding to an ideal axial crushing condition.

For a given input, the compliant mechanism design objective is to maximize the output displacement $d_{\text {out }}$. Assuming a fictitious or Page 2 of 13

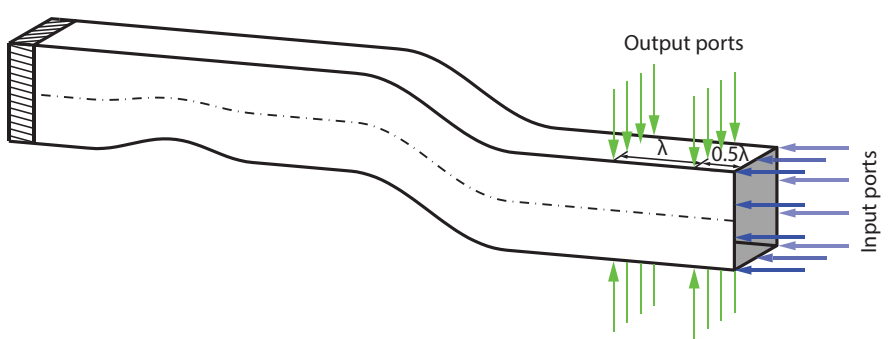

Figure 1. Locations of input and output ports for a thin-walled S-Tube following the wavelength $\lambda$ corresponding to the progressive buckling after an ideal axial crushing condition.

dummy unit-force vector $\mathbf{F}_{d}$ applied at the output ports-in the same direction of the desired displacement, $d_{\text {out }}$ can be expressed as:

$$
d_{\text {out }}=\left[\begin{array}{lllllll}
0 & \cdots & 0 & 1 & 0 & \cdots & 0
\end{array}\right]\left[\begin{array}{c}
\vdots \\
d_{\text {out }} \\
\vdots
\end{array}\right]=\mathbf{F}_{d}^{T} \mathbf{U},
$$

where $\mathbf{U}$ is the displacement vector corresponding to the input load and the term $\mathbf{F}_{d}^{T} \mathbf{U}$ is referred to as Mutual Potential Energy (MPE). For a discrete finite element model, MPE can be expressed as the sum of element quantities, i.e.,

$$
\mathrm{MPE}=\mathbf{F}_{d}^{T} \mathbf{U}=\sum_{i=1}^{n} \sigma_{d i}^{T} \varepsilon_{i},
$$

where $\sigma_{d}$ is the stress field produced by the dummy load and $\boldsymbol{\varepsilon}$ is the strain field due to the input load, and $n$ is the total number of elements used to discretize the structure. Thus, the topometry optimization problem for compliant mechanism design can be expressed to as

$$
\begin{array}{ll}
\text { find } & \mathbf{x}=\left[x_{1}, \cdots, x_{i} \cdots, x_{n}\right]^{T} \\
\text { minimize } & f(\mathbf{x})=-\mathrm{MPE}=-\mathbf{F}_{d}^{T} \mathbf{U}(\mathbf{x})=-\sum_{i=1}^{n} \sigma_{d i}^{T}(\mathbf{x}) \varepsilon_{i}(\mathbf{x}) \\
\text { subject to } & h(\mathbf{x})=\sum_{i=1}^{n} \rho_{i} A_{i} x_{i}-\bar{m}=0 \\
& \underline{x}_{i} \leq x_{i} \leq \bar{x}_{i}, i=1, \cdots, n,
\end{array}
$$

where $\mathbf{x}$ is the vector of design variables (i.e., element thickness), $\rho_{i}$ is the element material density, $A_{i}$ is the element area, $\bar{m}$ is the target mass, and $\underline{x}_{i}$ and $\bar{x}_{i}$ are the lower an upper limits with values $6 \times 10^{-4} \mathrm{~m}$ and $6 \times 10^{-3} \mathrm{~m}$, respectively.

\section{Design algorithm}

In this work, the Hybrid Cellular Automaton (HCA) algorithm [34] is used to solve the optimization problem in Eq. (3). The HCA algorithm uses a control strategy to find or approximate the problem's optimality conditions $[1,35]$. The problem's optimality conditions are 


$$
\begin{array}{lll}
\frac{\partial f\left(\mathbf{x}^{*}\right)}{\partial x_{i}}+\mu \frac{\partial h\left(\mathbf{x}^{*}\right)}{\partial x_{i}}=0 & \text { if } & x_{i}<x_{i}^{*}<\bar{x}_{i} \\
\frac{\partial f\left(\mathbf{x}^{*}\right)}{\partial x_{i}}+\mu \frac{\partial h\left(\mathbf{x}^{*}\right)}{\partial x_{i}} \geq 0 & \text { if } & x_{i}^{*}=\underline{x}_{i} \\
\frac{\partial f\left(\mathbf{x}^{*}\right)}{\partial x_{i}}+\mu \frac{\partial h\left(\mathbf{x}^{*}\right)}{\partial x_{i}} \leq 0 & \text { if } & x_{i}^{*}=\bar{x}_{i}
\end{array}
$$

where

$$
\frac{\partial h(\boldsymbol{x})}{\partial x_{i}}=\rho_{i} A_{i}
$$

and $\mu$ is the Lagrange multiplier associated with the equality constraint. The expression for the sensitivity of the objective function $f(\mathbf{x})$ depends on the interpolation function used to determine the material properties of intermediate materials $\left(\underline{x}_{i}<x_{i}^{*}<\bar{x}_{i}\right)$. For example, the Solid Isotropic Material with Penalization (SIMP) interpolation defines the stiffness matrix of intermediate materials as

$$
\mathbf{K}_{i}=E_{i} \mathbf{K}_{i}^{1}=\left[\underline{E}_{i}+x_{i}^{p}\left(E_{0}-\underline{E}_{i}\right)\right] \mathbf{K}_{i}^{1},
$$

where $\mathbf{K}_{i}^{\mathbf{1}}$ is the element stiffness matrix with unit Young's modulus $\left(E_{i}=1\right), E_{0}$ is the Young's modulus of the base material, $E_{i}$ is the minimum allowed value (e.g., $E_{i}=E_{0} \times 10^{-9}$ ), and $p$ is the penalization power. In this case, the sensitivity of the objective function $f(\mathbf{x})$ is given by

$$
\frac{\partial f(\mathbf{x})}{\partial x_{i}}=-p x_{i}^{p-1}\left(E_{0}-\underline{E}_{i}\right) \mathbf{U}_{d i}^{\mathrm{T}} \mathbf{K}_{i}^{1} \mathbf{U}_{i}=-p x_{i}^{p-1}\left(E_{0}-\underline{E}_{i}\right) \mathrm{MPE}_{i} .
$$

The optimality condition is obtained when the error between a mechanical stimulus and a set point is minimized (Figure 2). Defining the mechanical stimulus as $\mathrm{MPE}_{i}$, the set point is defined by

$$
s p_{i}=-\mu \frac{\rho_{i} A_{i}}{p x_{i}^{p-1}\left(E_{0}-\underline{E}_{i}\right)},
$$

and the error $e_{i}$ is given by

$$
e_{i}=-M P E_{i}-s p_{i}
$$

The value of $\mu$ is unknown but it can be found using a root finding algorithm for $h(\mathbf{x}, \mu)=0$, which ensures a feasible solution in every iteration [35]. The HCA iterative scheme is

$$
\mathrm{x}_{\mathrm{i}}^{\mathrm{t}+1}=\min \left\{\max \left\{\underline{\mathrm{x}}_{\mathrm{i}}, \mathrm{x}_{\mathrm{i}}^{\mathrm{t}}+\Delta \mathrm{x}_{\mathrm{i}}^{\mathrm{t}}\right\}, \overline{\mathrm{x}}_{\mathrm{i}}\right\}
$$

where

$$
\Delta x_{i}^{t}= \begin{cases}\kappa_{1} e_{i}^{t} & \text { for } t=1 \\ \kappa_{1} e_{i}^{t}+\kappa_{2} e_{i}^{t-1} & \text { for } t=2 \\ \kappa_{1} e_{i}^{t}+\kappa_{2} e_{i}^{t-1}+\kappa_{3} e_{i}^{t-2} & \text { for } t>2\end{cases}
$$

The constants $\kappa_{1}, \kappa_{2}$, and $\kappa_{3}$ are derived from a ProportionalIntegral-Derivative (PID) controller [1]. In our previous studies, the iterative scheme in Eq. (4) was used until no possible change in thickness was possible [28]. In this study, we apply a novel one iteration approach, which means our conceptual design is achieved by Page 3 of 13

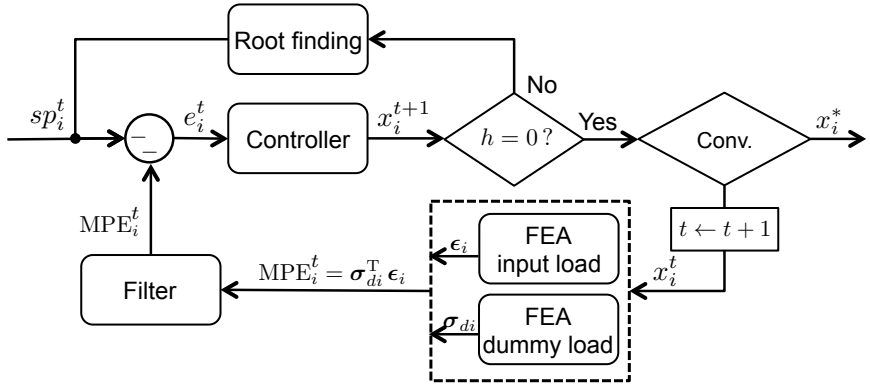

Figure 2. HCA algorithm described as a feedback control system operating in every discrete element comprising the design domain [1].

only one optimization iteration. The reasons why we only use one iteration instead of potentially hundreds of iterations are: (1) a reasonable conceptual design may be generated in one iteration, and (2) from past numerical studies, the result from one iteration has high-energy deformation mode - progressive folding.

\section{Crashworthiness indicators}

In order to evaluate a structure's crashworthiness, it is necessary to define measurable crashworthiness indicators. Commonly crashworthiness indicators include: internal energy, specific energy absorption, peak crushing force, and crash load efficiency. Considering the inelastic collision of a stationary mass $M$ (barrier) and a moving mass $m$ (thin-walled tubular component) traveling with a velocity $v_{0}$, where $M \gg m$. If the moving object comes to rest within a total crushing distance $\Delta \leq \Delta_{\max }$, where $\Delta_{\max }$ is the maximum crushing displacement after which no additional folds, then the initial kinetic energy is absorbed by the crushed thin-walled structure in the form of internal energy $U$. This is

$$
U(\Delta)=\int_{0}^{\Delta} P(\delta) d \delta=P_{m} \Delta=\frac{1}{2} m v_{0}^{2},
$$

where $P(\delta)$ is the crushing force, $\delta$ is the displacement, $P_{m}$ is the mean crushing force, and $\Delta$ is the maximum crushing distance is known under a general crushing mode, which is the case for finite element analysis, the mean crushing force is

$$
P_{m}=\frac{U(\Delta)}{\Delta}
$$

The specific energy absorption $U_{a}$ is energy absorbed per unit mass of the structure, that is

$$
U_{a}(\Delta)=\frac{U(\Delta)}{\rho V},
$$

where $V$ is the volume of the structure. The peak crushing force of the structure corresponds to

$$
P_{\max }=\max P(\delta) .
$$

Finally, the crash load efficiency is defined as the ratio of mean and peak crushing force, that is 


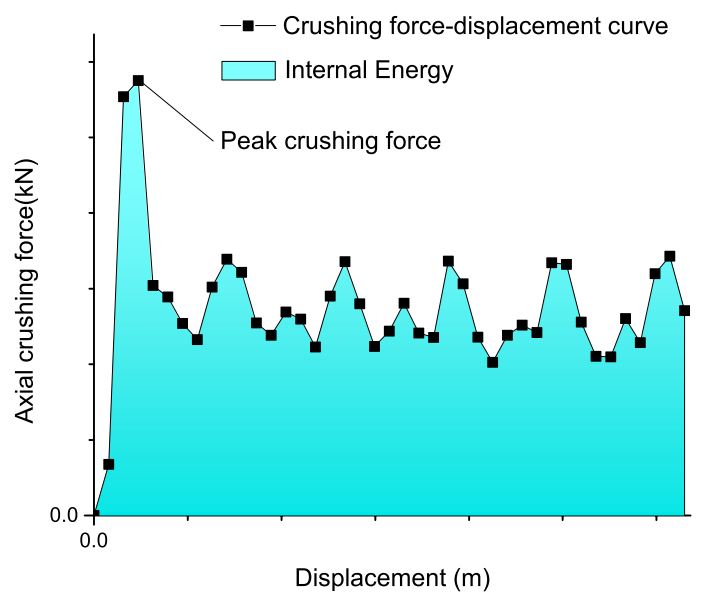

Figure 3. Axial crushing force-displacement curve of a typical thin-wall tubular structure.

$$
E_{c l}=\frac{P_{m}}{P_{\max }},
$$

which would ideally be driven to one by tailoring the shape and thickness of the thin-walled component (Figure 3).

\section{Numerical results}

The initial design of the problem (Figure 1) is a thickness uniformly distributed tube that satisfies the mass constraint, as shown on the left of Figure 4. The crash simulation at a certain time instance is shown on the right. From the simulation, two mechanical hinges exist on the S-shape. Figure 5 indicates the conceptual design obtained from maximizing MPE. The crash simulation of conceptual design indicates that, at the same time instance, structure is always progressively folded at the near to impact end and without any mechanical hinge. Table 1 shows the comparison of crashworthiness performance between the initial and conceptual (1-ITER) design. Notably, both structures have $\Delta=0.5 \mathrm{~m}$ and $m=9.25 \mathrm{~kg}$. In comparison to the initial design, the conceptual design shows $5.38 \%$ peak crushing force reduction, $13.15 \%$ specific energy absorption increments, and $17.95 \%$ crush load efficiency increment.
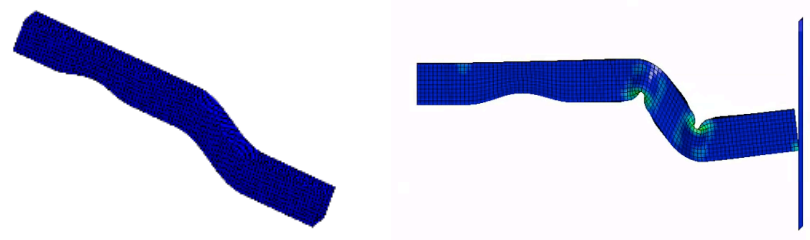

Figure 4. Initial design and its crash simulation.
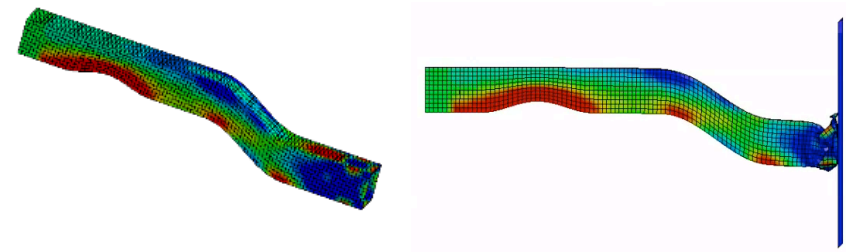

Figure 5. Conceptual design and its crash simulation.

Table 1. Initial and 1-ITER crashworthiness measurements.

\begin{tabular}{cccccc}
\hline & $U(k J)$ & $P_{m}(k N)$ & $P_{\max }(k N)$ & $U_{a}(k J / k g)$ & $E_{c l}$ \\
\hline Initial & 26.70 & 53.40 & 138.38 & 2.89 & 0.39 \\
1-ITER & 30.35 & 60.70 & 130.93 & 3.27 & 0.46 \\
\hline
\end{tabular}

\section{Design Parameterization}

The conceptual design is generated using over four thousand design variables. It is impractical to utilize general optimization schemes with such a high number of design variables. To overcome this problem, one major task of this investigation is to reduce the dimension of design space. Besides, the reduced number of design variables increases the manufacturability of the optimized design. In this work, we propose for the first time the use of unsupervised machine learning techniques to reduce the design space dimensionality. One promising unsupervised machine learning technique is K-means clustering.

\section{K-means clustering}

K-means, first used by James MacQueen in 1967 [36], remains as one of the most popular unsupervised machine learning techniques. In this method, given a set of observations $\mathbf{x} \in \mathbb{R}^{n}$ the algorithm aims to partition the $n$ observations into $K$ sets $\mathbf{S}=\left\{S_{1}, \ldots, S_{K}\right\}$ where $K \leq$ $n$. The objective is to minimize the within-cluster sum of squares defined as

$$
\begin{array}{ll}
\text { find } & \mu \in \mathbb{R}^{K} \\
\text { minimize } & J(\mu)=\sum_{k=1}^{K} \sum_{x_{i} \in S_{k}}\left\|x_{i}-\mu_{k}\right\|^{2},
\end{array}
$$

where $\mu_{k}$ is the mean of points in $S_{k}$. Commonly, an iterative refinement algorithm is used to perform K-means clustering [37]. The procedure of classifying data follows some simple steps as shown in Algorithm 1.

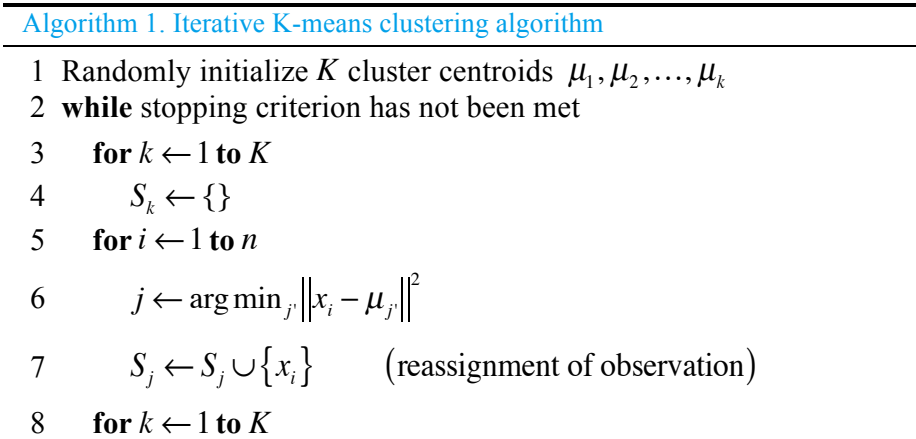


$9 \quad \mu_{k} \leftarrow \frac{1}{\left|S_{k}\right|} \sum_{x_{i} \in S_{k}} x_{i} \quad$ (recomputation of centroid)

10 return $\left\{\mu_{1}, \mu_{2}, \ldots, \mu_{K}\right\}$

The K-means clustering result is heavily dependent on the initial cluster centers (the first step in Algorithm 1), which is neither always global optimum nor repeatable. To increase the chance of K-means algorithm converging to the global minima, multiple restarts (typically 50-1000 times) can be used and clustering that gave lowest cost Eq. (11) can be picked. However this method is inefficient. As an alternative to the standard heuristic K-means algorithm, this investigation incorporates Ckmeans.1d.dp. Ckmeans.1d.dp is a $\mathrm{R}$ package that implements a dynamic programming algorithm to perform optimal one-dimensional K-means clustering [38]. It guarantees optimality and repeatability [39].

\section{Optimal K value}

In K-means, the number $K$ represents the number of means (clusters) one wants to partition for a set of observations. The choice of optimal $K$ value is always ambiguous, especially for not well-separated data sets. To enhance manufacturability as well as effective use of surrogate models later, the optimal $K$ value in this study is selected in the range of 2 and 20.

Using image-processing principles and assuming that the data is grouped into $K$ clusters, the K-means performance can be evaluated using the Silhouette function $\operatorname{Silh}(i)$ defined as

$$
\operatorname{Silh}(i)=\frac{b(i)-a(i)}{\max \{a(i), b(i)\}},
$$

where $a(i)$ is the average distance from the $i$-th observation to the other observations in the same cluster, $d(i)$ is he average distance from the $i$-th observation to the other observations in a difference cluster, and $b(i)=\min d(i)$. The average Silhouette width provides an evaluation of clustering validity. Notably, $-1 \leq \operatorname{Silh}(i) \leq 1$. If $\operatorname{Silh}(i)$ is close to 1 , then the data at point $i$ is an appropriate cluster. If $\operatorname{Silh}(i)$ is close to -1 , then the data at point $i$ is misclassified. Finally, if $\operatorname{Silh}(i)$ is close to 0 , then the data at point $i$ is on the boundary of two natural clusters. Figure 6 illustrates the mean Silhouette values with different numbers of clusters $K$ used to partition our conceptual design. The one with highest Silhouette value indicates the optimal $K$ value. Therefore, by using the Silhouette method, the optimal $K$ value is found to be 7 .

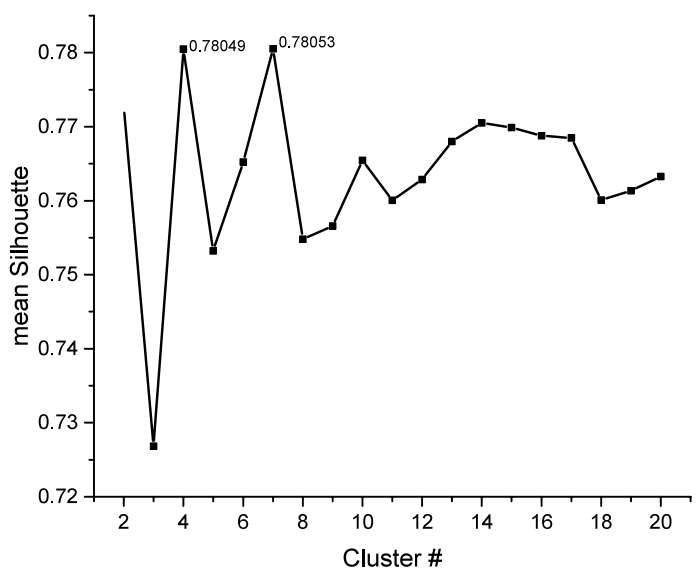

Figure 6. The average Silhouette width of different cluster number.

The effectiveness of the clustering process through K-means is evaluated through crashworthiness indicators. The comparisons of the crashworthiness between the conceptual design (1 ITER) and the parameterized designs are shown in Table 2. In these results, all structures have the same mass $m=9.25 \mathrm{~kg}$ and the same maximum crushing distance $\Delta=0.5 \mathrm{~m}$. In comparison to the conceptual design (1 ITER), the crashworthiness of the parameterized design K7 has $4.59 \%$ and $6.52 \%$ lower specific energy absorption as well as crush load efficiency, respectively. Therefore, this is not a good initial design for later optimization purposes.

To find the optimal $K$ value for our downstream purpose, the conceptual design is converted into a total of 19 initial designs with different $K$ values (from 2 to 20). Figure 7 shows the differences of specific energy absorption $\left(\delta U_{a}\right)$ and crush load efficiency $\left(\delta E_{c l}\right)$ along with the cluster number.

Table 2 Conceptual and parameterized designs crashworthiness measurements

\begin{tabular}{cccccc}
\hline & $U(k J)$ & $P_{m}(k N)$ & $P_{\max }(k N)$ & $U_{a}(k J / k g)$ & $E_{c l}$ \\
\hline 1 ITER & 30.35 & 60.70 & 130.93 & 3.27 & 0.46 \\
K2 & 33.48 & 66.97 & 144.22 & 3.61 & 0.46 \\
K3 & 17.45 & 34.90 & 93.03 & 1.88 & 0.38 \\
K4 & 26.75 & 53.50 & 142.32 & 2.89 & 0.38 \\
K5 & 27.16 & 54.32 & 140.12 & 2.93 & 0.39 \\
K6 & 30.23 & 60.46 & 143.29 & 3.27 & 0.42 \\
K7 & 28.92 & 57.84 & 134.68 & 3.12 & 0.43 \\
K8 & 28.71 & 57.41 & 150.34 & 3.10 & 0.38 \\
K9 & 27.65 & 55.30 & 134.22 & 2.99 & 0.41 \\
K10 & 31.53 & 63.06 & 131.96 & 3.41 & 0.48 \\
K11 & 30.82 & 61.63 & 125.55 & 3.33 & 0.49 \\
K12 & 31.65 & 63.30 & 122.45 & 3.42 & 0.52 \\
K13 & 30.97 & 61.93 & 122.94 & 3.35 & 0.50 \\
K14 & 26.69 & 53.39 & 143.02 & 2.88 & 0.37 \\
K15 & 28.48 & 56.96 & 140.24 & 3.08 & 0.41 \\
K16 & 28.29 & 56.58 & 137.05 & 3.06 & 0.41 \\
K17 & 28.01 & 56.02 & 137.98 & 3.03 & 0.41 \\
K18 & 31.65 & 63.30 & 143.06 & 3.42 & 0.44 \\
K19 & 31.78 & 63.56 & 132.65 & 3.43 & 0.48 \\
K20 & 30.92 & 61.84 & 128.76 & 3.34 & 0.48 \\
\hline & & & &
\end{tabular}




$$
\delta\langle\bullet\rangle_{\mathrm{K} k}=\frac{\langle\bullet\rangle_{1-\mathrm{ITER}}-\langle\bullet\rangle_{\mathrm{K} k}}{\langle\cdot\rangle_{1-\mathrm{ITER}}},
$$

where $\langle\bullet\rangle=U_{a}$ or $E_{c l}$, and $k=2,3, \ldots, 20$. The figure indicates that structure crashworthiness performances do not increase monotonically with the cluster number $K$. Among all the 19 initial designs, progressive folding disappears only on K2. Therefore, K2 should not be considered. Figure 8 plots the Pareto Front of SEA, $E_{c l}$, and $K$. In the figure, the ball indicates the non-dominated designs of SEA and $E_{c l}$, and the tetrahedron indicates the dominated designs. The optimal $K$ value is selected from the Pareto Front of SEA and $E_{c l}$ with the smallest number of $K$, i.e., $K=11$.

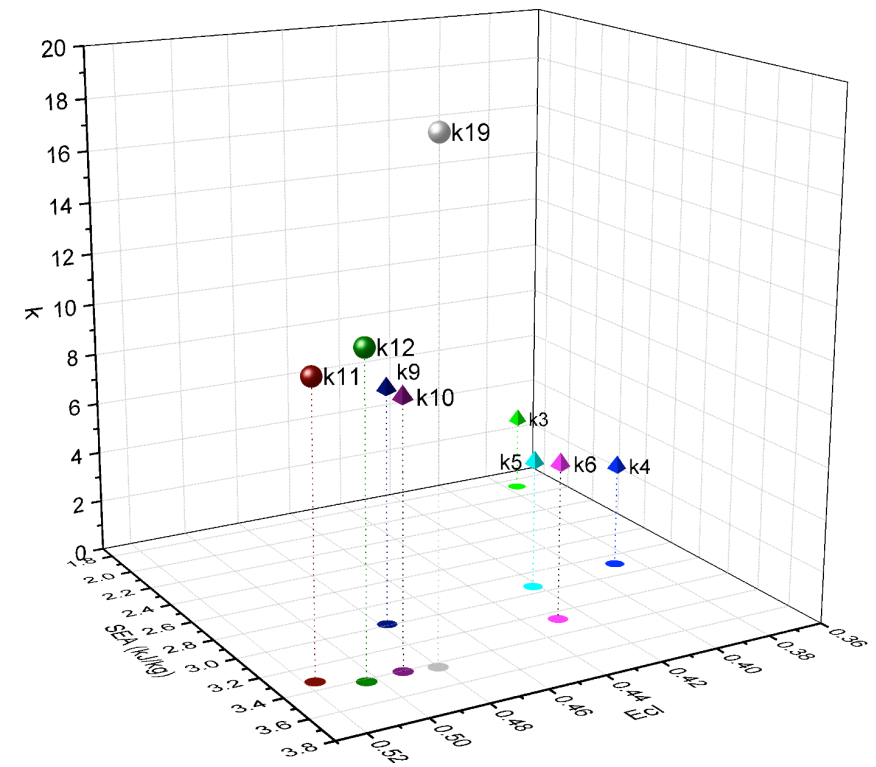

Figure 8. Pareto front of SEA, $E_{\mathrm{cl}}$ and $K$.

By selecting the proper value of $K$, the conceptual design is partitioned correspondingly. Figure 9 gives the initial 4000+ design variables that have been naturally broken into 11 groups using Kmeans.

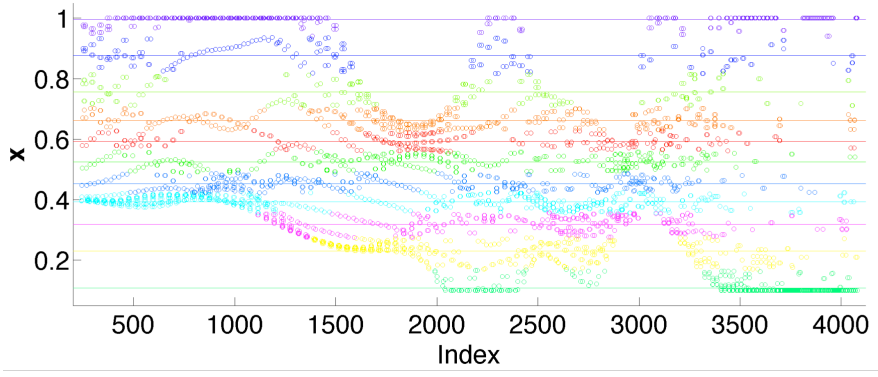

Figure 9. Example of K-means clustering that naturally separates a data set into 11 clusters. The horizontal axis is the index number of each point in $\mathrm{x}$. The 11 clusters are indicated using colored circles. The horizontal lines represent the means of each cluster.

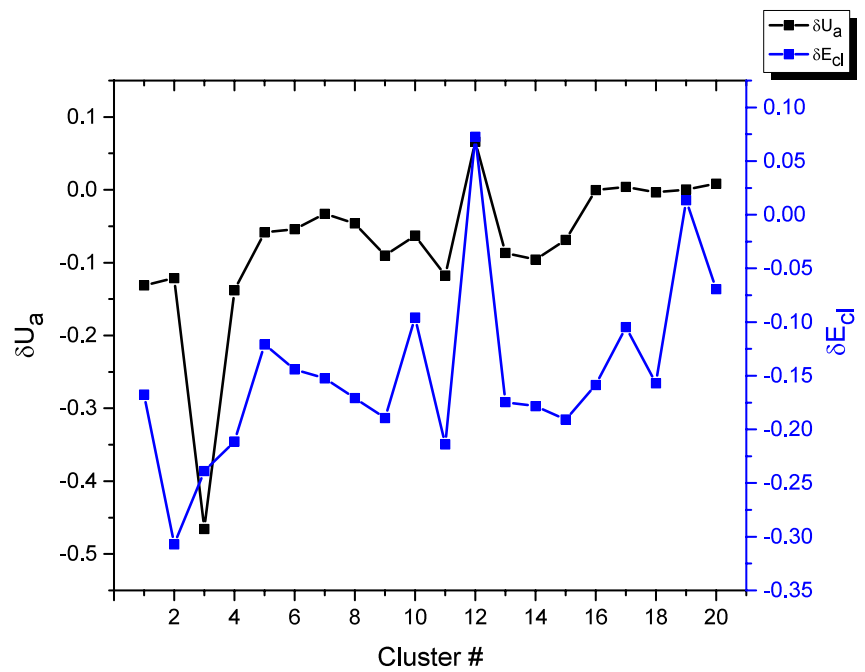

Figure 7. Differences in specific energy absorption and crush load efficiency between the conceptual design and the K-means clustered designs.

\section{Multi-objective Optimization}

From the previous step, the conceptual design has been clustered into 11 groups. The parametric optimization in this final step is to finding the thickness values that maximize crashworthiness indicators. Optimization problem formulations have traditionally included design objectives such as energy absorption [40], specific energy absorption and absorbed energy ratio [41], peak crushing force and specific energy absorption [42]. Let use consider all these objectives in a single multi-objective formulation.

\section{Problem formulation}

To find the optimal cluster thicknesses, multi-objective optimization formula is used in this investigation. Initially, the objectives are chosen as maximize specific energy absorption and crush load efficiency, which are the same metric used to determine the optimal $K$ value. Eqs. (6)-(10) indicate that crush load efficiency is proportional to the ratio of specific energy absorption and peak crushing force. Therefore, instead of maximizing two dependent objectives, we proposed a multi-objective problem with two independent, conflict objectives: maximizes specific energy absorption and minimizes peak crushing force. The optimization problem is defined as

$$
\begin{array}{ll}
\text { find } & \mu \in \mathbb{R}^{K}(K=11) \\
\text { maximize } & U_{a}(\mu) \\
\text { minimize } & P_{\max }(\mu) \\
\text { subject to } & \underline{\mu}_{k} \leq \mu_{k} \leq \bar{\mu}_{k}, \quad k=1, \ldots, K,
\end{array}
$$

where $U_{a}$ is the specific energy absorption as defined in Eq. (8). $P_{\text {max }}$ is the peak crushing force as defined in Eq. (9). $\underline{\mu}_{k}$ and $\bar{\mu}_{k}$ are the lower an upper thickness limits with values $6 \times 10^{-4} \mathrm{~m}$ and $6 \times 10^{-3} \mathrm{~m}$, respectively. The proposed multi-objective programming problem in Eq. (14) is solved by sequentially updating surrogate models. A flow chart of the proposed algorithm is shown in Figure 10, including sampling (design of experiments), crash simulation, fit 
surrogates, etc. Some key steps of the algorithm are described in the following.

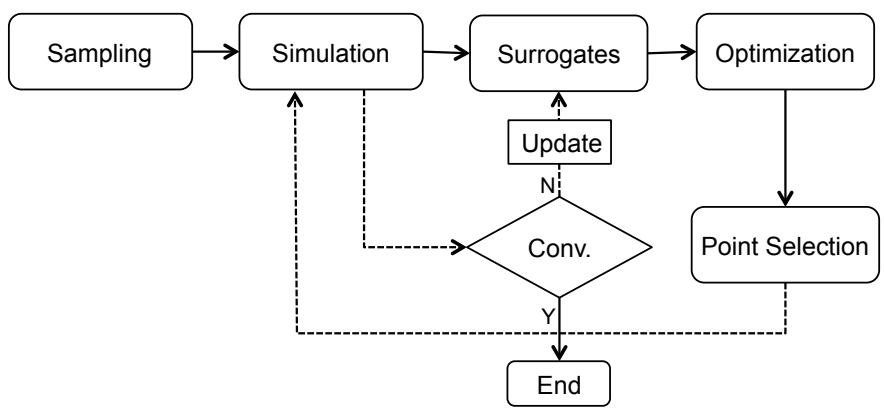

Figure 10. Sequential surrogates-based optimization algorithm.

\section{Design of experiments}

Design of experiments is the selection procedure for finding the points in the design space that must be analyzed. Many strategies can be used to sample the design points [43], e.g., the factorial, Doptimal, and Latin hypercube designs. This investigation utilizes Latin hypercube designs to sample the design points because they generate a set of samples that more precisely reflect the shape of a sampled distribution than pure random samples. These Latin hypercube designs are created by the MATLAB built-in function lhsdesign.

\section{Surrogates}

To reduce the computational cost of crashworthiness design problems, surrogate model - also called metamodel - methodologies have been often employed to replace the actual simulation models [44]. As a result of advances in computer throughput, researchers have incorporated multiple sophisticated and expensive metamodels [45-47]. However, the performances of surrogate models may vary from problem to problem, and the selection of surrogate models usually based on designers' experiences. This section provides the

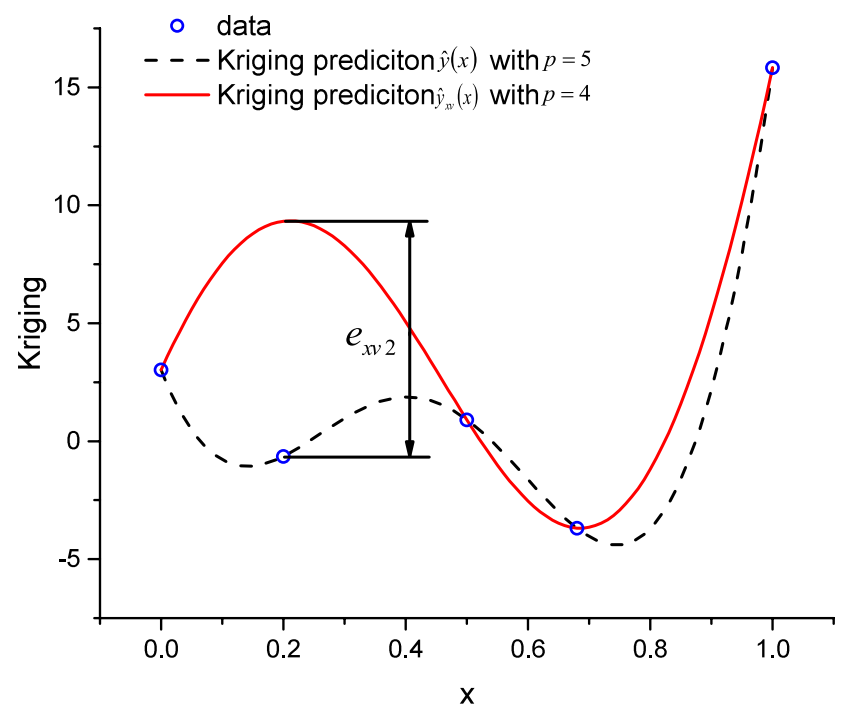

Figure 11. Cross validation error at the second sampled point $e_{x v 2}$, exemplified by fitting the function $y(x)=(6 x-2)^{2} \sin (2(6 x-2))$ with a Kriging model for $x=[0.0,0.2,0.5,0.68,1.0]^{\mathrm{T}}$ data points [2]. surrogates selecting procedure that implemented in this investigation.

\section{Basic surrogates and derived surrogates}

To find the best surrogate models suitable for our particular problem, this investigation studied three typical surrogate models, namely, Polynomial Response Surface (PRS) [43, 48], Radial Basis Function (RBF) [49-52], and Kriging (KRG) [53-55] as well as their different instances. Table A1 in the Appendix gives the details about the 24 different basic surrogates used during this investigation.

\section{Performance metrics}

To assess the surrogate performance, use of the sampling points may not be appropriate. Especially because RBF and KRG are interpolations, which means the surrogates can go through the sampling points themselves, making the tested values at the sampling points meaningless. Further, adding additional validation points requires more expensive crash simulations. For this reason, for comparing surrogates based on the data only at the $p$ points of the Design of Experiments (DOE), we use cross-validation errors [56]. A cross-validation error is the error at a sampling point when we fit the surrogate with all the other $p-1$ points except that point. When we fit the surrogate with this leave-one-out strategy, we obtain the vector of cross-validation error, $\mathbf{e}_{X V}$. This vector is also referred to as PREdiction Sum of Squares (PRESS) vector. Figure 11 illustrates computation of the cross validation errors for a Kriging surrogate.

The square root of the PRESS value is the estimator of the root mean square error, $e_{\mathrm{RMS}}[56]$ :

$$
\operatorname{PRESS}_{\mathrm{RMS}}=\sqrt{\frac{1}{p} \mathbf{e}_{x v}{ }^{T} \mathbf{e}_{x v}},
$$

and

$$
e_{\mathrm{RMS}}=\sqrt{\frac{1}{V} \int_{D}(\hat{y}(\mathbf{x})-y(\mathbf{x}))^{2} d \mathbf{x}},
$$

where $V$ is the volume of the design domain $D, y(\mathbf{x})$ and $\hat{y}(\mathbf{x})$ are the actual simulation and the surrogate prediction at the point $\mathbf{x}$, respectively. Since the PRESS $\mathrm{RMS}_{\text {is }}$ an estimator of the $e_{\mathrm{RMS}}$, one possible way of using multiple surrogates is to select the model with the smallest PRESS $_{\mathrm{RMS}}$ value.

\section{Selection based on PRESS RMS $_{\text {PI }}$}

Tukey boxplot [57] is used to graphically depict the PRESS $_{\mathrm{RMS}}$ for

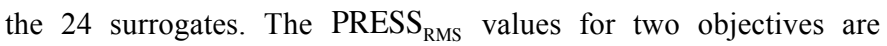
shown in Figure 12. The smallest PRESS $_{\text {RMS }}$ for Specific Energy Absorption is 0.51 , which is a fairly high value. By definition, specific energy absorption is the energy absorption (measured by internal energy) per unit mass, and structure mass can be calculated directly. Therefore, by evaluating the PRESS $_{\mathrm{RMS}}$ for Internal Energy, we decide to build a surrogate for internal energy instead of specific energy absorption. 
The surrogates (Table A1) that are selected based on PRESS $\mathrm{RMS}_{\mathrm{R}}$ value are: (14) krg-poly2-exp for internal energy, and (15) krg-poly2gauss for peak crushing force.

\section{Optimization and synthesis}

The multi-objective genetic algorithm serves as the global optimizer in this investigation. After optimum search, the Pareto front of $-U_{a}$ and $P_{\max }$ is generated. Figure 13 presents the non-dominated solutions about two conflict objectives of intermediate iteration. Among those non-dominated solutions, three points are selected for the update of the surrogate models: (i) the point whose $U_{a}$ is the largest, (ii) the point whose $P_{\max }$ is the smallest, and (iii) the point whose normalized distance to utopia point is the smallest.

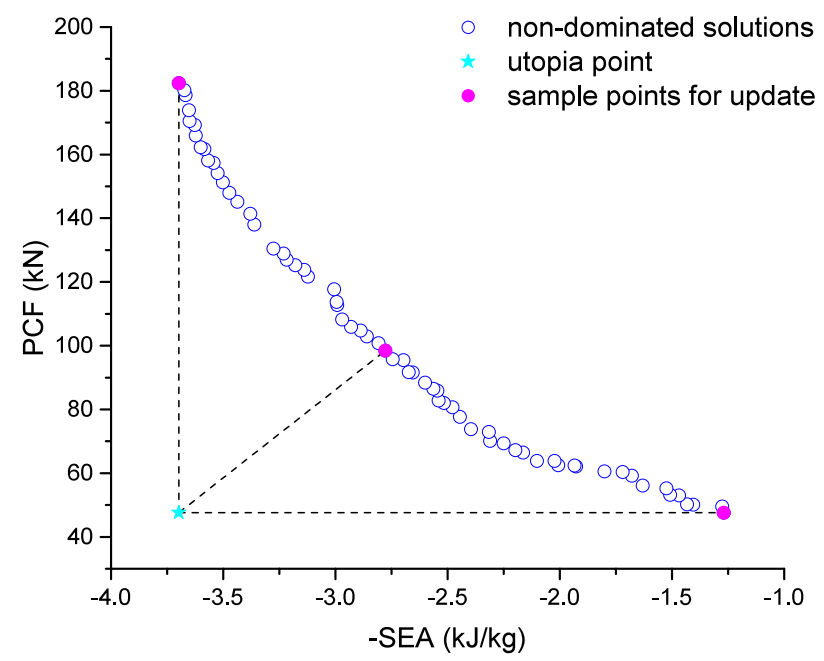

Figure 13. Illustration of point selection from Pareto front

\section{Convergence criteria}

Crash simulations of three newly selected designs are performed. The surrogate accuracy is calculated based on the following difference measure:

$$
\% \text { difference }=100 \times \frac{|\mathbf{y}(\mathbf{x})-\hat{\mathbf{y}}(\mathbf{x})|}{\mathbf{y}(\mathbf{x})} .
$$

If the maximum of the vector \%difference is within a relatively small number ( $=1 \%$ in our study), we conclude that the surrogate models are accurate enough, then the program end. Otherwise, we add the selected design to the sampling point set and reconstruct the surrogate models.

\section{Numerical results}

As expected, the Pareto-optimal set of the multi-objective programming problem in Eq. (14) dominates the initial conceptual design (1-ITER), which confirms the effectiveness of the proposed design strategy (Figure 14). Notably, both structures (1-ITER and K11) have been tested with the same maximum crushing distance $\Delta=0.5 \mathrm{~m}$. With a $99 \%$ reduction in the number of design variables and a $12.21 \%$ increment in mass, the Pareto-optimal solution (K11*)

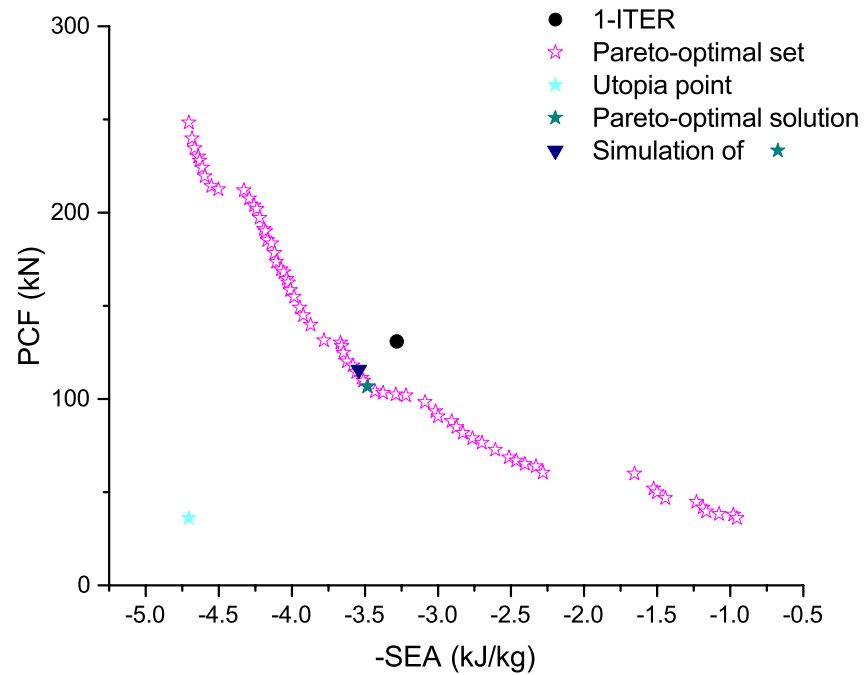

Figure 14. Pareto-optimal set of designed crashworthy thin-walled structure.

depicts better crashworthiness performance with $8.26 \%$ specific energy increment, $11.72 \%$ peak crushing force reduction, and $39.13 \%$ crush load efficiency increment (Table 3 ).

Table 3. Comparison of conceptual design (1-ITER) and parametric optimal design (K11)

\begin{tabular}{crccccc}
\hline & $m(k g)$ & $U(k J)$ & $P_{m}(k N)$ & $P_{\max }(k N)$ & $U_{a}(k J / k g)$ & $E_{c l}$ \\
\hline 1-ITER & 9.25 & 30.35 & 60.70 & 130.93 & 3.27 & 0.46 \\
K11 $^{*}$ & 10.38 & 36.75 & 73.50 & 115.58 & 3.54 & 0.64 \\
\hline
\end{tabular}

\section{Summary and Discussion}

This work introduces a new design algorithm for thin-walled crashworthy structures. The proposed design algorithm consists of three stages: conceptual thickness distribution, design parameterization, and multi-objective design optimization. With

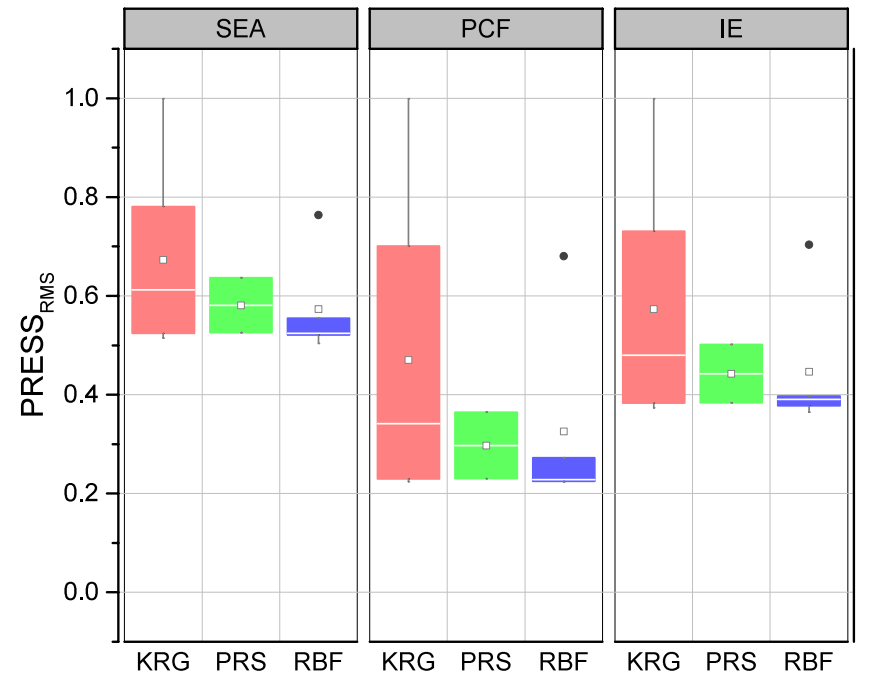

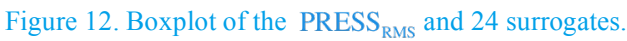


respect to the conceptual thickness distribution stage, most available publications aim to test geometries that promote progressive folding in thin-walled structures; in contrast, our work addresses the more fundamental problem consisting on the automatic generation of such geometries. The conceptual thickness distribution stage relies on our previous publications on the use of topology and topometry methods to design compliant, plastically deformable structures [27-29]. With respect to our previous work, the algorithm presented in this paper allows a significant reduction in the number of iterations: from potentially hundreds to only one. The resulting conceptual design depicts progressive folding and improves all crashworthiness measures with respect to the initial, uniform-thickness design.

During the design parameterization stage, the conceptual design is optimally segmented using the unsupervised machine learning technique K-means. The use of K-means allows reduction of the dimension of the design space from thousands to tens. Once the conceptual design is parameterized, the final structure is synthesized in the multi-objective design optimization stage. The proposed parametric optimization problem aims to find the cluster thickness values that maximize the specific energy absorption and minimize the peak crushing force. Due to the numerical cost of the simulation, the proposed optimization problem is solved with the use of surrogate models. The best surrogate model for each objective function is found within a set of surrogates according to the smallest prediction sum of squares error. The result of the sequential surrogates-based multi-objective programming problem is a set of Pareto optimal solutions that dominate the conceptual design with at least $99 \%$ fewer design variables.

Due to the limited number of thicknesses, this approach allows to synthesizing manufacturable thin-wall S-rail designs. However, one limitation is the inability to quantify progressive folding, which prevents from finding fully progressive folding structures. While all crashworthiness measures can be improved, the conceptual design is still superior to the optimized design with respect to progressive folding. Current research efforts aim to establish a progressive folding measure that to be incorporated in the multi-objective optimization algorithm.

\section{Acknowledgments}

Honda R\&D Americas supported this research effort. Any opinions, findings, conclusions, and recommendations expressed in this investigation are those of the writers and do not necessarily reflect the views of the sponsors.

\section{References}

1. Tovar, A. and Khandelwal, K., "Topology optimization for minimum compliance using a control strategy," Eng. Struct., 48(0): 674-682, 2013, doi:10.1016/j.engstruct.2012.12.008.

2. Keane, A.J., Sóbester, A., and Forrester, A.I.J., "Multi-fidelity optimization via surrogate modelling," Proceedings of the Royal Society A: Mathematical, Physical and Engineering Sciences, 463(2088): 3251-3269, 2007, doi:10.1098/rspa.2007.1900.

3. Kazanc1, Z. and Bathe, K.-J., "Crushing and crashing of tubes with implicit time integration," International Journal of Impact Engineering, 42: 80-88, 2012, doi:10.1016/j.ijimpeng.2011.10.003.

4. Jones, N. and Wierzbicki, T., "Structural crashworthiness and failure." Elsevier Science Publishers Ltd, 1-85166-969-8: 185166-969-8, 1993.

Page 9 of 13
5. Abah, L., Limam, A., and Dejeammes, M., "Effects of cutouts on static and dynamic behaviour of square aluminium extrusions," presented at Fifth international conference on structures under shock and impact, Greece, 1998.

6. Samer, F., Tarlochan, F., Khalili, P. et al., "Enhancement of energy absortion of thin walled hexagonal tube by using trigger mechanisms, "International Journal of Research in Engineering and Technology, 2(8): 109-116, 2013.

7. Shakeri, M., Mirzaeifar, R., and Salehghaffari, S., "New insights into the collapsing of cylindrical thin-walled tubes under axial impact load," Proceedings of the Institution of Mechanical Engineers, Part C: Journal of Mechanical Engineering Science, 221(8): 869-885, 2007.

8. Daneshi, G. and Hosseinipour, S., "Grooves effect on crashworthiness characteristics of thin-walled tubes under axial compression," Materials \& design, 23(7): 611-617, 2002.

9. Salehghaffari, S., Tajdari, M., Panahi, M. et al., "Attempts to improve energy absorption characteristics of circular metal tubes subjected to axial loading," Thin-Walled Structures, 48(6): 379$390,2010$.

10. Santosa, S.P., Wierzbicki, T., Hanssen, A.G. et al., "Experimental and numerical studies of foam-filled sections," Int. J. Impact Eng., 24(5): 509 - 534, 2000, doi:10.1016/S0734743X(99)00036-6.

11. Hou, S., Li, Q., Long, S. et al., "Crashworthiness design for foam filled thin-wall structures," Materials \& Design, 30(6): 2024 - 2032, 2009, doi:10.1016/j.matdes.2008.08.044.

12. Ghamarian, A., Zarei, H.R., Farsi, M.A. et al., "Experimental and Numerical Crashworthiness Investigation of the Empty and Foam-Filled Conical Tube with Shallow Spherical Caps," Strain, 49(3): 199-211, 2013, doi:10.1111/str.12028.

13. Zhang, Z., Liu, S., and Tang, Z., "Comparisons of honeycomb sandwich and foam-filled cylindrical columns under axial crushing loads," Thin-Walled Structures, 49(9): 1071 - 1079, 2011, doi:10.1016/j.tws.2011.03.017.

14. Sun, G., Li, G., Hou, S. et al., "Crashworthiness design for functionally graded foam-filled thin-walled structures," Mater. Sci. Eng. A, Struct. Mater., Prop. Microstruct. Process. (Switzerland), 527(7-8): $1911 \quad$ - 1919, 2010, doi:10.1016/j.msea.2009.11.022.

15. Yin, H., Wen, G., Hou, S. et al., "Multiobjective crashworthiness optimization of functionally lateral graded foam-filled tubes," Materials \& Design, 44(0): 414 - 428, 2013, doi:10.1016/j.matdes.2012.08.033.

16. Bendsøe, M.P. and Sigmund, O., "Topology Optimization: Theory, Methods and Applications." Springer, 2003.

17. Kang, B.-S., Park, G.-J., and Arora, J.S., "A review of optimization of structures subjected to transient loads," Structural and Multidisciplinary Optimization, 31(2): 81-95, 2006, doi:10.1007/s00158-005-0575-4.

18. Paik, J.K., Kim, B.J., Park, D.K. et al., "On quasi-static crushing of thin-walled steel structures in cold temperature: Experimental and numerical studies," International Journal of Impact Engineering, $\quad 38(1): \quad 13-28, \quad 2011$, doi:10.1016/j.ijimpeng.2010.08.004.

19. Mayer, R.R., Kikuchi, N., and Scott, R.A., "Application of Topological Optimization Techniques to Structural Crashworthiness," Int. J. Numer. Methods Eng., 39(8): 13831403, 1996, doi:10.1002/(sici)10970207(19960430)39:8<1383::aid-nme909>3.0.co;2-3.

20. Jung, D.Y. and Gea, H.C., "Topology optimization of nonlinear structures," Finite Elements in Analysis and Design, 40(11): 1417-1427, 2004, doi:10.1016/j.finel.2003.08.011. 
21. Soto, C.A., "Structural topology optimization for crashworthiness," International Journal of Crashworthiness, 9(3): 277-283, 2004, doi:10.1533/ijrc.2004.0288.

22. Wang, H., Ma, Z.-D., Kikuchi, N. et al., "Multi-domain multistep topology optimization for vehicle structure crashworthiness design," SAE Technical Paper 2004-01-1173, 2004.

23. Ortmann, C. and Schumacher, A., "Graph and heuristic based topology optimization of crash loaded structures," Structural and Multidisciplinary Optimization, 47(6): 839-854, 2013, doi:10.1007/s00158-012-0872-7.

24. Patel, N.M., Kang, B.S., Renaud, J.E. et al., "Crashworthiness Design Using Topology Optimization, " Journal of Mechanical Design, 131(6): 12, 2009, doi:061013 10.1115/1.3116256.

25. Mozumder, C., Renaud, J.E., and Tovar, A., "Topometry optimisation for crashworthiness design using hybrid cellular automata," Int. J. Veh. Des., 60(1/2): 100, 2012, doi:10.1504/ijvd.2012.049160.

26. Bandi, P., "Desing of crashworthy structures with controlled behavior in HCA framework," PhD thesis, Aerospace and Mechanical Engineering, University of Notre Dame, Notre Dame, Indiana,2012.

27. Bandi, P., Schmiedeler, J.P., and Tovar, A., "Design of Crashworthy Structures With Controlled Energy Absorption in the Hybrid Cellular Automaton Framework," J. Mech. Des., 135(9): 091002, 2013, doi:10.1115/1.4024722.

28. Shinde, S., Bandi, P., Detwiler, D. et al., "Structural Optimization of Thin-Walled Tubular Structures for Progressive Buckling Using Compliant Mechanism Approach," SAE Int. J. Passenger Cars Mech. Syst., 6(1): 109-120, 2013.

29. Bandi, P., Tovar, A., and Renaud, J., "Design of 2D and 3D Non-linear Compliant Mechanisms using Hybrid Cellular Automata," presented at 52nd AIAA/ASME/ASCE/AHS/ASC Structures, Structural Dynamics and Materials Conference, 2011.

30. Notghi, B., "Review of Crashworthiness Capability of Optimum S-shape Structure," Journal of Applied Sciences, 11(16): 28782882, 2011, doi:10.3923/jas.2011.2878.2882.

31. Liu, Y., Chu, S.J., and Viera, R., "Analysis of structural impact and crashworthiness using experimental, analytical and computational techniques: An overview and recent developments," International Journal of Vehicle Structures and Systems, 3(3): 144 - 153, 2011.

32. Sigmund, O., "On the design of compliant mechanisms using topology optimization," Mechanics of Structures and Machines, 25(4): 493-524, 1997, doi:10.1080/08905459708945415.

33. Saxena, A. and Ananthasuresh, G.K., "On an optimal property of compliant topologies," Struct. Multidiscip. Optim., 19(1): 3649, 2000, doi: $10.1007 / \mathrm{s} 001580050084$.

34. Tovar, A., Patel, N.M., Niebur, G.L. et al., "Topology optimization using a hybrid cellular automation method with local control rules," Journal of Mechanical Design, Transactions of the ASME, 128(6): 1205-1216, 2006, doi:10.1115/1.2336251.

35. Tovar, A., Patel, N.M., Kaushik, A.K. et al., "Optimality conditions of the hybrid cellular automata for structural optimization," AIAA Journal, 45(3): 673-683, 2007, doi:10.2514/1.20184

36. MacQueen, J.B., "Some Methods for classification and Analysis of Multivariate Observations," in 5-th Berkeley Symposium on Mathematical Statistics and Probability. 1967, University of California Press. p. 281-297.

37. MacKay, D., "Information Theory, Inference, and Learning Algorithms." Cambridge University Press, 2003.

38. Song, J. and Wang, H., Ckmeans.1d.dp: Optimal k-means clustering for one-dimensional data (Version 3.02), 2014.

Page 10 of 13
39. Wang, H. and Song, M.Z., "Ckmeans.1d.dp: optimal k-means clustering in one dimension by dynamic programming," The $R$ Journal, 3(2): 29-33, 2011.

40. Jung, D. and Gea, H.C., "Design of an energy-absorbing structure using topology optimization with a multimaterial model," Struct. Multidiscip. Optim., 32(3): 251-257, 2006, doi:10.1007/s00158-006-0011-4.

41. Huang, X., Xie, Y.M., and Lu, G., "Topology optimization of energy-absorbing structures," Int. J. Crashworthiness, 12(6): 663-675, 2007, doi:10.1080/13588260701497862.

42. Hou, S., Li, Q., Long, S. et al., "Multiobjective optimization of multi-cell sections for the crashworthiness design," Int. J. Impact Eng., 35(11): 1355-1367, 2008, doi:10.1016/j.ijimpeng.2007.09.003.

43. Myers, R.H. and Montgomery, D.C., "Response Surface Methodology. Process and Product Optimization using Designed Experiments." Wiley, New York, 1995.

44. Yang, S. and Qi, C., "Multiobjective optimization for empty and foam-filled square columns under oblique impact loading," Int. J. Impact Eng., 54: 177 - 191, 2013 , doi:10.1016/j.ijimpeng.2012.11.009.

45. Bi, J., Fang, H., Wang, Q. et al., "Modeling and optimization of foam-filled thin-walled columns for crashworthiness designs," Finite Elem. Anal. Des., 46(9): 698-709, 2010, doi:10.1016/j.finel.2010.03.008.

46. Song, X., Sun, G., Li, G. et al., "Crashworthiness optimization of foam-filled tapered thin-walled structure using multiple surrogate models," Struct. Multidiscip. Optim., 47(2): 221-231, 2012, doi:10.1007/s00158-012-0820-6.

47. Yin, H., Wen, G., Liu, Z. et al., "Crashworthiness optimization design for foam-filled multi-cell thin-walled structures," ThinWalled Structures, 75: 8-17, 2014, doi:10.1016/j.tws.2013.10.022.

48. Box, G., Hunter, W., and Hunter, J., "Statistics for experimenters." Wiley, New York, 1978.

49. Gutmann, H., "A radial basis function method for global optimization," J. Global Optim., 19(3): 201-227, 2001, doi:10.1023/A:1011255519438

50. Sun, G., Li, G., Gong, Z. et al., "Radial basis functional model for multiobjective sheet metal forming optimization," Eng. Optim., $\quad 12(43)$ : $1351-1366, \quad 2011$, doi:10.1080/0305215X.2011.557072.

51. Regis, R. and Shoemaker, C., "Constrained global optimization of expensive black box Functions using radial basis functions," J. Global Optim., 31(1): 153-171, 2005, doi:10.1007/s10898004-0570-0.

52. Mullur, A.A. and Messac, A., "Metamodeling using extended radial basis functions: a comparative approach," Engineering with Computers, 21(3): 203-217, 2005, doi:10.1007/s00366005-0005-7.

53. Sacks, J., Welch, W.J., Mitchell, T.J. et al., "Design and analysis of computer experiments," Stat Sci, 4(4): 409-423, 1989, doi:10.1214/ss/1177012413.

54. Simpson, T.W., Peplinski, J., Koch, P.N. et al., "On the Use of Statistics in Design and the Implications for Deterministic Computer Experiments," presented at Design Engineering Technical, Sacramento, CA, United State, 1997.

55. Lophaven, S., Nielsen, H., and Sondergaard, J., "DACE - a MATLAB kriging toolbox," in Tech. Rep. IMM-TR-2002-12. 2002: Technical University of Denmark, Denmark.

56. Viana, F.A.C., Haftka, R.T., and Steffen, V., "Multiple surrogates: how cross-validation errors can help us to obtain the best predictor," Struct. Multidiscip. Optim., 39(4): 439-457, 2009, doi:10.1007/s00158-008-0338-0. 
57. Frigge, M., Hoaglin, D.C., and Iglewicz, B., "Some Implementations of the Boxplot," The American Statistician, 43(1): 50, 1989, doi:10.2307/2685173.

58. Viana, F., SURROGATES Toolbox User's Guide (Version 3.0), Gainesville, FL, USA, 2011.

59. Jekabsons, G., RBF: Radial Basis Function interpolation for MATLAB/OCTAVE (Version 1.1), Riga Technical University, Latvia, 2009. 
Abbreviations

Design of Experiments

Equivalent Static Loads

Hybrid Cellular Automata

Kriging

MPE

PCF

PID

PRESS

Mutual Potential Energy

Peak Crushing Force

Proportional-Integral-

Derivative
PRS

$\mathrm{RBF}$

SEA

SIMP

PREdiction Sum of Squares
Polynomial Response

Surface

Radial Basis Function

Specific Energy Absorption

Solid Isotropic Material with Penalization 


\section{Appendix}

Table A1. Setup for the set of used surrogates

\begin{tabular}{|c|c|c|}
\hline Surrogates & Fitting function $\hat{y}(\mathbf{x})$ & Approximation / Correlation Kernel \\
\hline \multicolumn{3}{|c|}{ Kriging (approximation-correlation) } \\
\hline (1) krg-poly0-cubic & \multirow{17}{*}{$\begin{aligned} \hat{\beta}+\mathbf{r}^{T}(\mathbf{x}) \mathbf{R}^{-1}(\mathbf{y}-\mathbf{f} \hat{\beta}) & \\
\mathbf{R}(\theta, \omega, x) & =\prod_{i=1}^{n} R_{i}\left(\theta, d_{i}\right) \\
d_{i} & =\omega_{i}-x_{i}\end{aligned}$} & poly0: $f_{1}(x)=1$ \\
\hline (2) krg-poly0-exp & & poly1: $f_{1}(x)=1, f_{2}(x)=x_{1}, \ldots, f_{n+1}(x)=x_{n}$ \\
\hline (3) krg-poly0-gauss & & poly2: $f_{1}(x)=1$ \\
\hline (4) krg-poly0-lin & & $f_{2}(x)=x_{1}, \ldots, f_{n+1}(x)=x_{n}$ \\
\hline (5) krg-poly0-spherical & & $f_{n+2}(x)=x_{1}^{2}, \ldots, f_{2 n+1}(x)=x_{1} x_{n}$ \\
\hline (6) krg-poly0-spline & & $f_{2 n+2}(x)=x_{2}^{2}, \ldots, f_{3 n}(x)=x_{2} x_{n}$ \\
\hline (7) krg-poly1-cubic & & cubic: $R_{i}\left(\theta, d_{i}\right)=1-3 \xi_{i}^{2}+2 \xi_{i}^{3}$ \\
\hline (8) krg-poly1-exp & & $\xi_{i}=\min \left\{1, \theta_{i}\left|d_{i}\right|\right)$ \\
\hline (9) krg-poly1-gauss & & $\exp : R_{i}\left(\theta, d_{i}\right)=e^{-\theta_{i}\left|d_{i}\right|}$ \\
\hline $\begin{array}{l}\text { (10) krg-polyl-lin } \\
\text { (11) kro-polyl-spherical }\end{array}$ & & \\
\hline (12) krg-poly1-spline & & gauss: $R_{i}\left(\theta, d_{i}\right)=e^{-\theta_{i} d_{i}^{2}}$ \\
\hline \multicolumn{2}{|l|}{ (13) krg-poly2-cubic } & \\
\hline (14) krg-poly2-exp & & $\operatorname{lin}: R_{i}\left(\theta, d_{i}\right)=\max \left\{0,1-\theta_{i}\left|d_{i}\right|\right\}$ \\
\hline (15) krg-poly2-gauss & & spherical: $R_{i}\left(\theta, d_{i}\right)=1-1.5 \xi_{i}^{2}+0.5 \xi_{i}^{3}$, \\
\hline (16) krg-poly2-lin & & $\xi_{i}=\min \left\{1, \theta_{i}\left|d_{i}\right|\right)$ \\
\hline \multicolumn{2}{|l|}{ (17) krg-poly2-spherical } & \\
\hline (18) krg-poly2-spline & & $\varsigma\left(\xi_{i}\right)= \begin{cases}1-15 \xi_{i}^{2}+30 \xi_{i}^{3} & \text { for } 0 \leq \xi_{i} \leq 0.2 \\
1.25\left(1-\xi_{i}\right)^{3} & \text { for } 0.2<\xi_{i}<1 \\
0 & \text { for } \xi_{i} \geq 1\end{cases}$ \\
\hline \multicolumn{3}{|c|}{ Polynomial Response Surface } \\
\hline (19) prs-poly1 & $\beta_{0}+\sum_{i=1}^{n} \beta_{i} x_{i}$ & N/A \\
\hline (20) prs-poly2 & $\beta_{0}+\sum_{i=1}^{n} \beta_{i} x_{i}+\sum_{i<j} \beta_{i j} x_{i} x_{j}+\sum_{i=1}^{n} \beta_{i i} x_{i}^{2}$ & N/A \\
\hline \multicolumn{3}{|l|}{ Radial Basis Function } \\
\hline (21) rbf-MQ & \multirow{4}{*}{$\begin{array}{r}\sum_{i=1}^{n} \lambda_{i} \varphi\left(r\left(\mathbf{x}_{i}, \mathbf{x}\right)\right) \\
r\left(\mathbf{x}_{i}, \mathbf{x}\right)=\left\|\mathbf{x}-\mathbf{x}_{i}\right\|\end{array}$} & Multiquadic: $\varphi(r)=\sqrt{1+(\varepsilon r)^{2}}$ \\
\hline (22) rbf-IMQ & & Inverse multiquadic: $\varphi(r)=\frac{1}{\sqrt{1+(\varepsilon r)^{2}}}$ \\
\hline (23) rbf-TPS & & Thin plate spline: $\varphi(r)=r^{2} \ln (r)$ \\
\hline (24) rbf-G & & Gaussian: $\varphi(r)=e^{-(\varepsilon r)}$ \\
\hline
\end{tabular}

The DACE[55], SURROGATES[58], and RBF[59] toolboxes were used to run the Kriging, polynomial response surface, radial basis function, respectively. 